Document downloaded from:

http://hdl.handle.net/10251/160426

This paper must be cited as:

Bonet Solves, JA.; Mengestie, T.; Worku, M. (2019). Dynamics of the Volterra-type integral and differentiation operators on generalized Fock spaces. Results in Mathematics. 74(4):115. https://doi.org/10.1007/s00025-019-1123-7

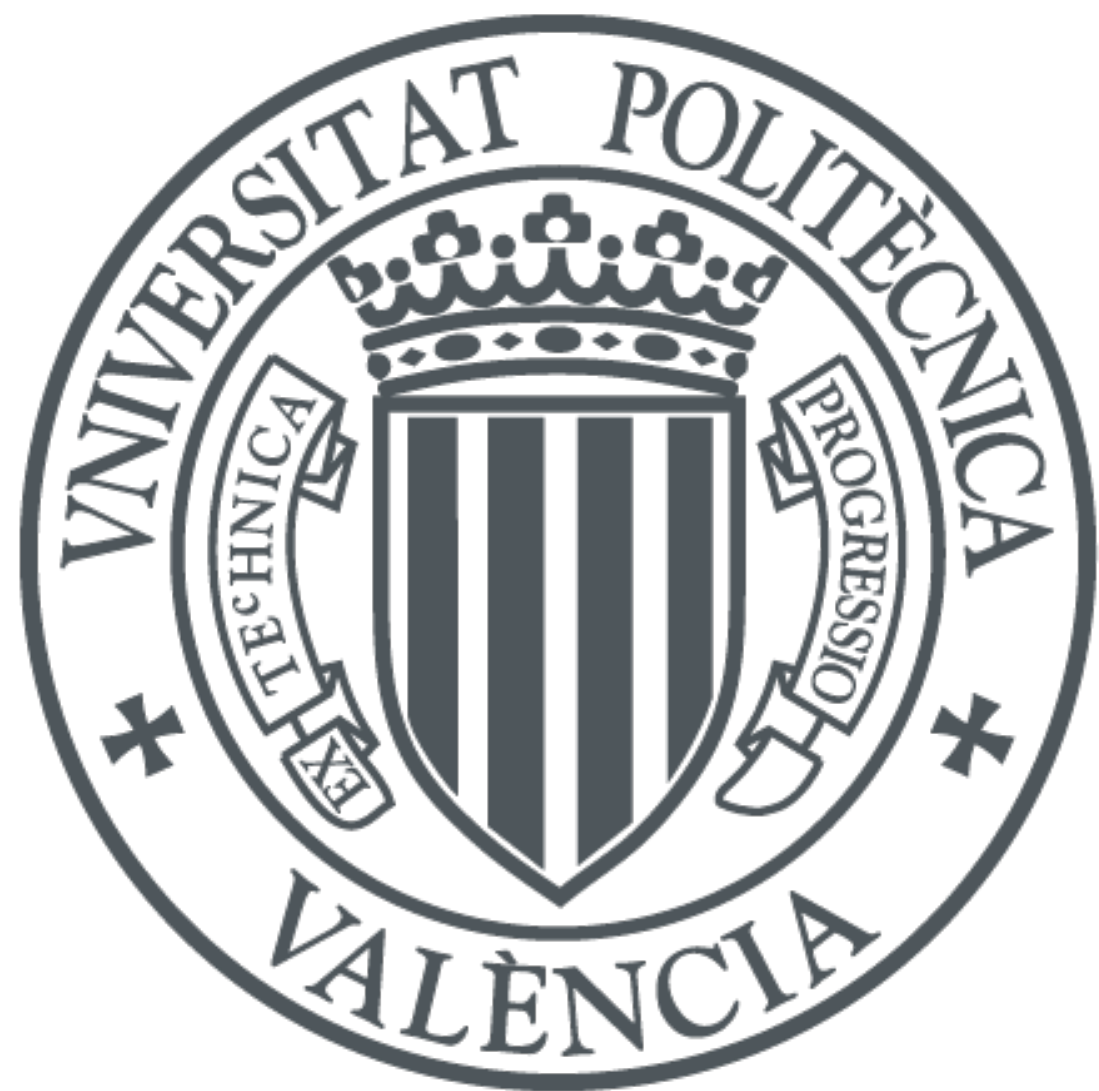

The final publication is available at

https://doi.org/10.1007/s00025-019-1123-7

Copyright Springer-Verlag

Additional Information 


\title{
DYNAMICS OF THE VOLTERRA-TYPE INTEGRAL AND DIFFERENTIATION OPERATORS ON GENERALIZED FOCK SPACES
}

\author{
JOSÉ BONET*, TESFA MENGESTIE, AND MAFUZ WORKU
}

\begin{abstract}
Various dynamical properties of the differentiation and Volterratype integral operators on generalized Fock spaces are studied. We show that the differentiation operator is always supercyclic on these spaces. We further characterize when it is hypercyclic, power bounded and uniformly mean ergodic. We prove that the operator satisfies the Ritt's resolvent condition if and only if it is power bounded and uniformly mean ergodic. Some similar results are obtained for the Volterra-type and Hardy integral operators.
\end{abstract}

\section{INTRODUCTION}

For holomorphic functions $f$ and $g$, the differentiation operator $D f=f^{\prime}$ and the Volterra-type integral operator $V_{g} f(z)=\int_{0}^{z} g^{\prime}(w) f(w) d w$ are classical objects in operator theory, function spaces and differential equations. Many of their basic properties including boundedness, compactness and spectra have been extensively studied when acting on several function spaces over various domains; see for example $[1,5,6,8,10,11,13,20,21,22,23]$ and the references therein. Understanding the dynamical structures of these operators is another important and basic problem in operator theory. The main purpose of this paper is to study such structures on generalized Fock spaces $\mathcal{F}_{(\alpha, m)}^{p}$. We are especially interested in identifying their various forms of cyclicity, power boundedness, and uniform mean ergodic properties.

Let us first set the generalized Fock spaces. For $m, \alpha>0$ and $0<p<\infty$, the spaces $\mathcal{F}_{(\alpha, m)}^{p}$ consist of all entire functions $f$ for which

$$
\|f\|_{(p, \alpha, m)}^{p}=\int_{\mathbb{C}}|f(z)|^{p} e^{-p \alpha|z|^{m}} d A(z)<\infty
$$

\footnotetext{
$1 *$ Corresponding author

2010 Mathematics Subject Classification. Primary: 47B38, 30H20; Secondary: 46E15, 47A16, 47A35.

Key words and phrases. Generalized Fock spaces, power bounded, uniformly mean ergodic, Volterra-type integral operator, differential operator, Hardy operator, supercyclic, hypercyclic, cyclic, Ritt's resolvent condition.

The research of the first author was partially supported by the research projects MTM201676647-P and GV Prometeo 2017/102 (Spain).

The research of the third author is supported by ISP project, Addis Ababa University, Ethiopia.
} 
where $d A$ denotes the usual Lebesgue area measure on $\mathbb{C}$. Note that the norm can be rewritten in terms of the integral mean $M_{p}^{p}(f, r)=\int_{0}^{2 \pi}\left|f\left(r e^{i t}\right)\right|^{p} \frac{d t}{2 \pi}$ as

$$
\|f\|_{(p, \alpha, m)}^{p}=2 \pi \int_{0}^{\infty} M_{p}^{p}(f, r) r e^{-p \alpha r^{m}} d r .
$$

We now introduce a few relevant notations needed in the rest of the article. The expression $U(z) \lesssim V(z)$ (or equivalently $V(z) \gtrsim U(z)$ ) means that there is a constant $C$ such that $U(z) \leq C V(z)$ holds for all $z$ in the set in question. We write $U(z) \simeq V(z)$ if both $U(z) \lesssim V(z)$ and $V(z) \lesssim U(z)$.

The norms of the monomials will play an important role in studying the dynamical properties of both the differentiation and integral operators on the spaces $\mathcal{F}_{(\alpha, m)}^{p}$. Thus, we estimate them using Stirling's formulas,

$$
n ! \simeq \sqrt{n} n^{n} e^{-n} \text { and } \Gamma(x+1) \simeq \sqrt{x} x^{x} e^{-x}, x>0,
$$

where $\Gamma$ denotes the Gamma function, and we get

$$
\begin{gathered}
\left\|z^{n}\right\|_{(p, \alpha, m)}=\left(2 \pi \int_{0}^{\infty} r^{p n} e^{-p \alpha r^{m}} r d r\right)^{1 / p}=\frac{\left(2 \pi \Gamma\left(\frac{p n}{m}+\frac{2}{m}\right)\right)^{1 / p}}{m(p \alpha)^{\frac{n}{m}+\frac{2}{m p}}} \\
\simeq \frac{(p n+2-m)^{\frac{n}{m}+\frac{2}{m p}-\frac{1}{2 p}}}{(p \alpha)^{\frac{n}{m}+\frac{2}{m p}} m^{\frac{n}{m}+\frac{2}{m p}+\frac{1}{2 p}} e^{\frac{n}{m}+\frac{2}{m p}-\frac{1}{p}}} \simeq\left(\frac{n}{m e \alpha}\right)^{\frac{n}{m}+\frac{2}{m p}-\frac{1}{2 p}} .
\end{gathered}
$$

From the preceding estimate we have in particular, for all $n \in \mathbb{N}$,

$$
\left\|z^{n}\right\|_{(p, \alpha, 1)} \simeq n ! \alpha^{-n} n^{\frac{3-p}{2 p}} .
$$

Next, we recall some definitions related to iterates of an operator. Given a Banach space $X$, we denote by $\mathcal{L}(X)$ the space of continuous linear operators $T$ on $X$. An operator $T \in \mathcal{L}(X)$ is said to be hypercyclic if there exists a vector $x$ in $X$ such that its orbit, $\left\{T^{n} x ; n \in \mathbb{N}_{0}=\{0\} \cup \mathbb{N}\right\}$, is dense in $X$. The operator is called cyclic if the linear span of an orbit is dense in $X$, and supercyclic whenever a projective orbit, $\left\{\lambda T^{n} x ; n \in \mathbb{N}_{0}, \lambda \in \mathbb{C}\right\}$, is dense in $X$. Obviously, hypercyclicity is a stronger property than supercyclicity which in turn is stronger than cyclicity. Good references on this subject are $[3,16]$.

An operator $T \in \mathcal{L}(X)$ is said to be power bounded if there exists a positive number $M$ such that $\left\|T^{n}\right\| \leq M$ for all $n \in \mathbb{N}_{0}$. The operator $T$ is called quasinilpotent if $\lim _{n \rightarrow \infty}\left\|T^{n}\right\|^{1 / n}=0$. It is said to be mean ergodic if there exists an operator $P \in \mathcal{L}(X)$ such that

$$
P x:=\lim _{n \rightarrow \infty} \frac{1}{n} \sum_{k=1}^{n} T^{k} x, x \in X
$$

exists in $X$. If the convergence is in the operator norm, then $T$ is called uniformly mean ergodic. The standard references about mean ergodic operators are the books of Krengel [18] and Yosida [29]. 


\section{Dynamics of the DifFerentiation operator on $\mathcal{F}_{(\alpha, m)}^{p}$}

The differentiation operator $D$ has been studied on Banach spaces of analytic functions by several authors. Harutyunyan and Lusky [17] identified conditions under which the operator becomes bounded when acting between weighted spaces of holomorphic functions endowed with the supremum norm; see also [1]. Bonet [8] studied various dynamical properties of the operator on these weighted spaces, and the study was continued jointly with Beltrán, Bonilla and Fernández in [6, 10]. Later in 2014, Beltrán [5] studied the dynamics of the operator on a wider class of generalized weighted Bergman spaces. On the other hand, the operator is also known to act in unbounded way in several functional spaces. For instance in [28] Ueki showed its unboundedness on the classical growth type Fock spaces. Mengestie and Ueki [23] verified its unboundedness on all classical Fock spaces and generalized Fock spaces where the weight function grows faster than the Gaussian weight function $|z|^{2} / 2$. The same conclusion was later drawn in [21] on the Fock-Sobolev spaces which are typical examples of generalized Fock spaces with weight function growing slower than the Gaussian function. Inspired by all these, Mengestie [20] asked the question of how fast should the weight function need to grow in order that the corresponding generalized Fock spaces support a continuous differentiation operator. He further considered the spaces $\mathcal{F}_{(1, m)}^{p}$ and showed that the weight function should actually grow much slower than the classical Gaussian function. More specifically, it was proved that the operator $D$ is bounded on $\mathcal{F}_{(1, m)}^{p}, 0<p<\infty$, if and only if $m \leq 1$, and compact if and only if $m<1$. See also [5, Section 5]. In this section, we continue those lines of research and investigate the dynamical behaviour of the operator $D$ on $\mathcal{F}_{(\alpha, m)}^{p}$.

Proposition 2.1. Let $1 \leq p<\infty$ and let the differentiation operator $D$ be bounded on $\mathcal{F}_{(\alpha, m)}^{p}$. Then

(i) $D$ is hypercyclic on $\mathcal{F}_{(\alpha, m)}^{p}$ if and only if either $m=1$ and $\alpha>1$ or $m=1, \alpha=1$ and $p>3$.

(ii) $D$ is supercyclic and hence cyclic on $\mathcal{F}_{(\alpha, m)}^{p}$.

Proof. (i) First note that since $D$ is bounded, $m \leq 1$, as can be seen from (1.2). In addition, since no compact operator is hypercyclic on a non zero complex Banach space [3, Corollary 1.22], it follows that $D$ is not hypercyclic on $\mathcal{F}_{(\alpha, m)}^{p}$ whenever $m<1$. On the other hand, for $m=1$, using the relation in (1.3), we have

$$
\liminf _{n \rightarrow \infty} \frac{\left\|z^{n}\right\|_{(p, \alpha, 1)}}{n !} \simeq \liminf _{n \rightarrow \infty} \frac{n^{\frac{3}{2 p}}}{\alpha^{n} \sqrt{n}}=\left\{\begin{array}{l}
0, \text { for } \alpha=1 \text { and } p>3 \text { or } \alpha>1 \\
1, \text { for } \alpha=1 \text { and } p=3 \\
\infty, \text { for } \alpha<1 \text { or } \alpha=1 \text { and } p<3
\end{array}\right.
$$

Then, by Theorem 5.2 of [5], $D$ is hypercyclic if and only if either $\alpha=1$ and $p>$ 3 or $\alpha>1$.

(ii) For this part, we follow the arguments used in the proof of [8, Proposition 2.7]. Since for each $n \in \mathbb{N}$ the monomial $z^{n}$ belongs to the kernel $\operatorname{Ker} D^{n+1}$ of $D^{n+1}$, 
the generalized kernel set

$$
G_{\text {Ker }}:=\bigcup_{n=0}^{\infty} \operatorname{Ker} D^{n}
$$

contains all the polynomials. Since the polynomials are dense in $\mathcal{F}_{(\alpha, m)}^{p}$, it follows that $G_{\text {Ker }}$ is dense in $\mathcal{F}_{(\alpha, m)}^{p}$. Moreover, the range of the operator $D$ contains polynomials and therefore it is dense in $\mathcal{F}_{(\alpha, m)}^{p}$. Then our conclusion follows after an application of [4, Corollary 3.3].

We note that the proof of part (i) depends on the hypercyclicity criterion due to Bés and Peris [7], where the original idea goes back to the work of Kitai in her Ph.D. thesis [16, Theorem 3.4]. The aforementioned Theorem 5.2 of [5] ensures that $D$ is hypercyclic on generalized Bergman spaces if and only if it satisfies the hypercyclicity criterion. This was further shown to be equivalent to a condition like

$$
\liminf _{n \rightarrow \infty}(n !)^{-1}\left\|z^{n}\right\|_{(p, \alpha, 1)}=0
$$

which remains valid in our setting. Similarly, the proof of part (ii) was based on a density condition in [4]. This condition is equivalent to the known supercyclicity criterion; see [4, Lemma 3.1]. Therefore, $D$ satisfies the supercyclicity criterion if and only it is supercyclic. We note that not all supercyclic operators satisfy this criterion; see [14] for an example.

Having completely identified conditions under which $D$ is hypercyclic, we next consider the question of when $D$ can be topologically mixing on $\mathcal{F}_{(\alpha, m)}^{p}$. Recall that an operator $T$ on a Banach space $X$ is topologically mixing if for every pair of non-empty open subsets $U$ and $V$ of $X$, there exists an $N \in \mathbb{N}$ such that $T^{n}(U) \cap V \neq \emptyset$ for all $n \geq N$. Note that topologically mixing is a stronger operator theoretic condition than hypercyclicity in general. Following the discussions above and the arguments used back in the proof of Theorem 2.4 and Corollary 2.6 of [8], the differentiation operator $D$ is topologically mixing on $\mathcal{F}_{(\alpha, m)}^{p}$ whenever it is hypercyclic.

Now we investigate when the differentiation operator is power bounded and uniformly mean ergodic.

Theorem 2.2. Let $1 \leq p<\infty$ and the differentiation operator $D$ be bounded on $\mathcal{F}_{(\alpha, m)}^{p}$. Then the following statements are equivalent.

(i) $D$ is power bounded and uniformly mean ergodic on $\mathcal{F}_{(\alpha, m)}^{p}$.

(ii) Either $m<1$ or $m=1$ and $\alpha<1$.

Proof. We first show that (ii) implies $(i)$. Since $D$ is bounded, we have $m \leq 1$. If $m<1$, then the operator is compact and by [20, Theorem 1.2], its spectrum $\sigma(D)$ contains only the zero element. By the spectral formula, there exist $\delta<1$ and $N \in \mathbb{N}$ such that

$$
\left\|D^{n}\right\| \leq \delta^{n} \text { for all } n \geq N
$$


and therefore, $D$ is power bounded and uniformly mean ergodic in this case. For the case when $m=1$ and $\alpha<1$, arguing as in (5.3) in [5, Proposition 5.9], we get

$$
\left\|D^{k} f\right\|_{(p, \alpha, 1)}^{p} \leq \frac{(k !)^{p} e^{p \alpha k}}{k^{p k}}\|f\|_{(p, \alpha, 1)}^{p} .
$$

Using this along with Stirling formula (1.1), we deduce

$$
\left\|D^{k}\right\| \lesssim \frac{k^{\frac{p}{2}}}{e^{(1-\alpha) k}} \rightarrow 0
$$

as $k \rightarrow \infty$ for $\alpha<1$, and hence the operator is power bounded in this case as well. Now, applying the estimate in (2.2),

$$
\left\|\frac{1}{n} \sum_{k=1}^{n} D^{k}\right\| \leq \frac{1}{n} \sum_{k=1}^{n}\left\|D^{k}\right\| \lesssim \frac{1}{n} \sum_{k=1}^{n} \frac{k^{\frac{p}{2}}}{e^{(1-\alpha) k}} \rightarrow 0
$$

as $n \rightarrow \infty$, from which it follows that $D$ is uniformly mean ergodic.

Now we show that (i) implies (ii). Assume that $m=1$. Using exponential functions $e_{\beta}(z)=e^{\beta z},|\beta|<\alpha$, we get $\overline{D(0, \alpha)} \subset \sigma(D)$, where $D(0, \alpha)$ is a disc with center 0 and radius $\alpha$. Thus, 1 is an accumulation point of $\sigma(D)$ whenever $\alpha \geq 1$. Since $D$ is assumed to be power bounded, we apply [15, Theorem 3.16] (see also [18, Theorem 2.7]), to get that the operator cannot be uniformly mean ergodic in this case. Therefore, we must have $\alpha<1$.

\section{Dynamics of the VolterRa-type integral operator on $\mathcal{F}_{(\alpha, m)}^{p}$}

In this section we investigate the dynamics of Volterra-type integral operators

$$
V_{g} f(z)=\int_{0}^{z} g^{\prime}(w) f(w) d w .
$$

Various aspects of the operator which includes boundedness, compactness, and spectra have been well studied in large class of function spaces; see for examples $[11,12,13,21,24,23]$ and the references therein. Much less is known about its dynamical and mean ergodic properties, except in the special case when the symbol $g$ is the identity map. The fact that the iterates of the operator involve multiple integrals makes it difficult to get best possible estimates of the norms. In this paper, we begin the study of the dynamical properties of $V_{g}$ on the spaces $\mathcal{F}_{(\alpha, m)}^{p}$. It was shown [13] (see also [12]), and in [11] for $p=\infty$, that $V_{g}$ is bounded on $\mathcal{F}_{(\alpha, m)}^{p}$ if and only if $g$ is a complex polynomial of degree $l$ not bigger than $m$ $(l \leq m)$, and $V_{g}$ is compact in this space if and only if the degree $l$ of $g$ is strictly smaller than $m$ or $m$ is not a positive integer.

Proposition 3.1. Let $1 \leq p<\infty$. Let $V_{g}$ be bounded on $\mathcal{F}_{(\alpha, m)}^{p}$ and hence $g(z)=a_{l} z^{l}+a_{l-1} z^{l-1}+\ldots+a_{1} z+a_{0}, l \leq m$. Then

(i) $V_{g}$ is not supercyclic on $\mathcal{F}_{(\alpha, m)}^{p}$ and hence not hypercyclic.

(ii) If $g(z)=a z^{l}+b, a \neq 0$, then $V_{g}$ is cyclic if and only if $l=1$. 
Proof. (i) Since $V_{g} f(0)=0$ for every $f$ in $\mathcal{F}_{(\alpha, m)}^{p}$, the projective orbit of $f$ under $V_{g}$ contains only functions that vanish at zero. Thus, it cannot be dense in $\mathcal{F}_{(\alpha, m)}^{p}$, which implies that $V_{g}$ is not supercyclic on $\mathcal{F}_{(\alpha, m)}^{p}$ and hence not hypercyclic either.

(ii) If $l=1$, then $\left(V_{g}\right)^{n}(\mathbf{1})(z)=\frac{a^{n} z^{n}}{n !}$. Hence

$$
\left\{\left(V_{g}\right)^{n}(\mathbf{1}), n \geq 0\right\}=\left\{1, a z, \frac{a^{2} z^{2}}{2}, \cdots, \frac{a^{n} z^{n}}{n !}, \cdots\right\} .
$$

The linear span of the latter is known to be dense in $\mathcal{F}_{(\alpha, m)}^{p}$.

If $l>1$ and $f$ belongs to $\mathcal{F}_{(\alpha, m)}^{p}$, using a functional that annihilates the $(l-1)$ th Taylor polynomial of $f$, together with the monomials $z^{k}, k \geq l$, we conclude that $f$ cannot be a cyclic vector for $V_{g}$.

The class of Volterra-type integral operators include the classical integration operator $J f(z)=\int_{0}^{z} f(w) d w$ in particular when $g(z)=z$. By $[13,23],[1]$ or $[24$, Lemma 1.1], it follows that $J$ is bounded on $\mathcal{F}_{(\alpha, m)}^{p}$ if and only if $m \geq 1$ and compact if and only if $m>1$. These conditions are opposite to the corresponding conditions for the differentiation operator $D$ except when $m=1$, in which case both $J$ and $D$ are bounded. Clearly $D J f=f$ and $J D f(z)=f(z)-f(0)$ for all $z \in \mathbb{C}$ and $f$ in $\mathcal{F}_{(\alpha, m)}^{p}$. Observe that while $D$ is supercyclic, there exists no vector whose projective orbit under $J$ is dense in $\mathcal{F}_{(\alpha, m)}^{p}$.

To state our next main result, we first recall some definitions. Denote by $\mathcal{H}(\mathbb{C})$ the set of entire functions on $\mathbb{C}$. For $r \geq 0$ and each $f \in \mathcal{H}(\mathbb{C})$, set

$$
M_{\infty}(f, r)=\sup _{|z|=r}|f(z)|,
$$

and define the growth type space $\mathcal{F}_{(\alpha, m)}^{\infty}$ as the space of functions $f \in \mathcal{H}(\mathbb{C})$ such that

$$
\|f\|_{(\infty, \alpha, m)}=\sup _{r>0} e^{-\alpha r^{m}} M_{\infty}(f, r)<\infty .
$$

The estimate corresponding to (1.2) becomes

$$
\left\|z^{n}\right\|_{(\infty, \alpha, m)} \simeq\left(\frac{n}{m e \alpha}\right)^{\frac{n}{m}}
$$

For $m=1$, the space $\mathcal{F}_{(\alpha, 1)}^{p}$ is denoted by $B_{p, p}(\alpha)$ in [5]. To simplify the notation below, we write the symbol $g(z)=a_{l} z^{l}+a_{l-1} z^{l-1}+\ldots+a_{1} z+a_{0}$, with $l \leq m$, as $g=g_{l}+g_{l-1}$ where $g_{l}(z)=a_{l} z^{l}$ and $g_{l-1}(z)=a_{l-1} z^{l-1}+\ldots+a_{1} z+a_{0}$. Then the operators on $\mathcal{F}_{(\alpha, m)}^{\infty}$ satisfy

$$
V_{g}=V_{g_{l}}+V_{g_{l-1}}
$$

of which $V_{g_{l-1}}$ is always compact and quasi-nilpotent.

Following [2], for each $\lambda \in \mathbb{C}, a \in \mathbb{C}$ and $m \in \mathbb{N}$, the operator $K_{\lambda}$ is defined on $\mathcal{H}(\mathbb{C})$ by

$$
K_{\lambda} f(z)=a m e^{\lambda z^{m}} \int_{0}^{z} e^{-\lambda w^{m}} w^{m-1} f(w) d w=a m z^{m} \int_{0}^{1} e^{\lambda z^{m}\left(1-t^{m}\right)} t^{m-1} f(t z) d t
$$


Thus, when $\lambda=0 m=l \in \mathbb{N}$ and $a=a_{l}$, then $K_{\lambda}$ is just the Volterra-type integral operator $V_{g_{l}}$.

Lemma 3.2. Let $m \geq 1, m \in \mathbb{N}$.

(i) If $|\lambda|<\alpha$, then the operator $K_{\lambda}$ is continuous on $\mathcal{F}_{(\alpha, m)}^{\infty}$ with operator norm $\left\|K_{\lambda}\right\| \leq \frac{|a|}{\alpha-|\lambda|}$.

(ii) If $l=m \in \mathbb{N}$, then $V_{g_{l}}$ is continuous on $\mathcal{F}_{(\alpha, m)}^{\infty}$ and its operator norm satisfies $\left\|V_{g_{l}}\right\| \leq \frac{\left|a_{l}\right|}{\alpha}$.

Proof. (i) For $r>0$, we have

$$
\begin{array}{r}
e^{-\alpha r^{m}} M_{\infty}\left(K_{\lambda} f, r\right)=e^{-\alpha r^{m}}|a| m r^{m} \int_{0}^{1} e^{|\lambda| r^{m}\left(1-t^{m}\right)} t^{m-1} M_{\infty}(f, r t) d t \\
=|a| m r^{m} \int_{0}^{1} t^{m-1} e^{-\alpha(t r)^{m}} e^{(\alpha-|\lambda|) r^{m}\left(t^{m}-1\right)} M_{\infty}(f, r t) d t \\
\leq|a| m r^{m}\|f\|_{(\infty, \alpha, m)} \int_{0}^{1} t^{m-1} e^{(\alpha-|\lambda|) r^{m}\left(t^{m}-1\right)} d t \leq \frac{|a|}{\alpha-|\lambda|}\|f\|_{(\infty, \alpha, m)} .
\end{array}
$$

Therefore

$$
\left\|K_{\lambda} f\right\|_{(\infty, \alpha, m)}=\sup _{r>0} e^{-\alpha r^{m}} M_{\infty}\left(K_{\lambda} f, r\right) \leq \frac{|a|}{\alpha-|\lambda|}\|f\|_{(\infty, \alpha, m)} .
$$

(ii) This is a direct consequence of part (i) for $l=m, a=a_{l}$ and $\lambda=0$.

Theorem 3.3. Let $1 \leq p \leq \infty, m \geq 1$ and $l \in \mathbb{N}$. Assume that the operator $V_{g}$ is bounded on $\mathcal{F}_{(\alpha, m)}^{p}$, with $g(z)=g_{l}(z)+g_{l-1}(z), g_{l}(z)=a_{l} z^{l}, l \leq m$. Then

(i) If $m>1,1 \leq p \leq \infty$ and $l<m$, then $V_{g}$ is compact, quasi-nilpotent, hence power bounded, and uniformly mean ergodic on $\mathcal{F}_{(\alpha, m)}^{p}$.

(ii) If $l=m \in \mathbb{N}$ and $p=\infty$, then $V_{g}$ is power bounded if and only if $\left|a_{l}\right| \leq \alpha$.

(iii) If $l=m \in \mathbb{N}, p=\infty$ and $\left|a_{l}\right| \leq \alpha$, then $V_{g_{l}}$ is uniformly mean ergodic if and only if $\left|a_{l}\right|<\alpha$.

(iv) If $l=m \in \mathbb{N}$ and $1 \leq p<\infty$ and $V_{g}$ is power bounded, then $\left|a_{l}\right| \leq \alpha$.

(v) If $l=m \in \mathbb{N}$ and $1 \leq p<\infty$ and $V_{g_{l}}$ is power bounded and uniformly mean ergodic, then $\left|a_{l}\right|<\alpha$.

Proof. (i) From results in $[11,23]$ for $p=\infty$ and in [13] for $1 \leq p<\infty$, the operator $V_{g}$ is compact on $\mathcal{F}_{(\alpha, m)}^{p}$ for all $1 \leq p \leq \infty$, since $l<m$. Moreover, by $[9$, Theorem $]$ and $[12$, Theorem 1$]$, we have $\sigma\left(V_{g}\right)=\{0\}$, hence $V_{g}$ is quasinilpotent. By the spectral radius formula, there exist $\beta<1$ and $N \in \mathbb{N}$ such that

$$
\left\|V_{g}^{n}\right\| \leq \beta^{n} \text { for all } n \geq N
$$

This shows that $V_{g}$ is power bounded.

The operator $V_{g}$ is also uniformly mean ergodic in this case. Indeed, an application of (3.2) yields, for some $C>0$ depending on $N$,

$$
\frac{1}{n}\left\|\sum_{k=1}^{n} V_{g}^{k}\right\| \leq \frac{1}{n} \sum_{k=1}^{n}\left\|V_{g}^{k}\right\| \leq \frac{C}{n}+\frac{\beta}{n(1-\beta)} \rightarrow 0 \text { as } n \rightarrow \infty \text {. }
$$


(ii) Now $p=\infty$ and $l=m \in \mathbb{N}$. Assume that $\left|a_{l}\right| \leq \alpha$. By Lemma 3.2 (ii) we have, for each $n \in \mathbb{N}$,

$$
\left\|V_{g_{l}}^{n}\right\| \leq\left|a_{l}\right|^{n} / \alpha^{n} \leq 1
$$

This implies that $V_{g_{l}}$ is power bounded. Since the sum of a power bounded operator and a quasi-nilpotent operator is power bounded, we conclude that $V_{g}$ is power bounded.

Conversely, suppose that $V_{g}$ is power bounded. Since $V_{g_{l-1}}$ is quasi-nilpotent by part (i), we get that $V_{g_{l}}$ is power bounded. However, for each positive integer $n$, a straightforward integral computation gives

$$
V_{g_{l}}^{n}(\mathbf{1})(z)=\frac{l^{n}\left(a_{l}\right)^{n} z^{l n}}{\prod_{j=1}^{n}(j l)}=\frac{\left(a_{l}\right)^{n} z^{l n}}{n !}
$$

from which and (3.1) we have

$$
\left\|V_{g_{l}}^{n}\right\| \geq \frac{\left\|V_{g_{l}}^{n} \mathbf{1}\right\|_{(\infty, \alpha, l)}}{\|\mathbf{1}\|_{(\infty, \alpha, l)}} \simeq \frac{\left|a_{l}\right|^{n}}{n !(\alpha e l)^{n}}(n l)^{n} \simeq \frac{\left|a_{l}\right|^{n}}{\alpha^{n}} \frac{1}{\sqrt{n}} \rightarrow \infty
$$

as $n \rightarrow \infty$ whenever $\left|a_{l}\right|>\alpha$, a contradiction. Thus $\left|a_{l}\right| \leq \alpha$.

(iii) In this part we assume $p=\infty, l=m \in \mathbb{N}$ and $\left|a_{l}\right| \leq \alpha$. We first suppose that $\left|a_{l}\right|<\alpha$. By Lemma 3.2 we have, for each $n \in \mathbb{N}$,

$$
\left\|V_{g_{l}}^{n}\right\| \leq\left|a_{l}\right|^{n} / \alpha^{n}
$$

Hence

$$
\frac{1}{n}\left\|\sum_{k=1}^{n} V_{g_{l}}^{k}\right\| \leq \frac{1}{n} \sum_{k=1}^{n}\left\|V_{g_{l}}^{k}\right\| \leq \frac{\left|a_{l}\right|}{n\left(\alpha-\left|a_{l}\right|\right)} \rightarrow 0 \text { as } n \rightarrow \infty,
$$

and $V_{g_{l}}$ is uniformly mean ergodic.

Conversely, suppose that $V_{g_{l}}$ is uniformly mean ergodic and that $\left|a_{l}\right| \leq \alpha$. Part (ii) implies that $V_{g_{l}}$ is power bounded. From [9] it follows that the spectrum $\sigma\left(V_{g_{l}}\right)=\left\{\lambda \in \mathbb{C}:|\lambda| \leq \frac{\left|a_{l}\right|}{\alpha}\right\}$. Thus, if $\left|a_{l}\right|=\alpha$, then 1 is an accumulation point of $\sigma\left(V_{g_{l}}\right)$. By [15, Theorem 3.16] (see also [18, Theorem 2.7]), the operator $V_{g_{l}}$ is not uniformly mean ergodic. Therefore $\left|a_{l}\right|<\alpha$.

(iv) If $l=m \in \mathbb{N}$ and $1 \leq p<\infty$ and $V_{g}$ is power bounded, then $V_{g_{l}}$ is power bounded since $V_{g_{l-1}}$ is quasi-nilpotent by part (i). Now, an integral computation again gives, for each positive integers $k$ and $n$,

$$
V_{g_{l}}^{n}\left(z^{k}\right)=\frac{\left(a_{l}\right)^{n} l^{n} z^{l n+k}}{\prod_{j=1}^{n}(j l+k)}
$$

and hence

$$
\begin{aligned}
&\left\|V_{g_{l}}^{n}\right\| \gtrsim \limsup _{k \rightarrow \infty} \frac{\left\|V_{g_{l}}^{n}\left(z^{k}\right)\right\|_{(p, \alpha, l)}}{\left\|z^{k}\right\|_{(p, \alpha, l)}}=\limsup _{k \rightarrow \infty} \frac{\left|a_{l}\right|^{n} l^{n}\left\|z^{n l+k}\right\|_{(p, \alpha, l)}}{\prod_{j=1}^{n}(j l+k)\left\|z^{k}\right\|_{(p, \alpha, l)}} \\
& \geq \limsup _{k \rightarrow \infty} \frac{\left|a_{l}\right|^{n} l^{n}\left\|z^{n l+k}\right\|_{(p, \alpha, l)}}{(n l+k)^{n}\left\|z^{k}\right\|_{(p, \alpha, l)}},
\end{aligned}
$$


where the last inequality follows since

$$
\prod_{j=1}^{n}(j l+k)=e^{\sum_{j=1}^{n} \log (j l+k)} \leq e^{n \log (n l+k)}=(n l+k)^{n} .
$$

Applying the norm estimate in (1.2),

$$
\frac{\left\|z^{n l+k}\right\|_{(p, \alpha, l)}}{\left\|z^{k}\right\|_{(p, \alpha, l)}} \simeq \frac{(n l+k)^{n}}{(e \alpha)^{n}}\left(1+\frac{n l}{k}\right)^{\frac{k}{l}+\frac{2}{p l}-\frac{1}{2 p}}
$$

and plugging this in (3.3) and making further simplifications

$$
\begin{aligned}
\left\|V_{g_{l}}^{n}\right\| \geq \frac{\left|a_{l}\right|^{n}}{\alpha^{n}} \limsup _{k \rightarrow \infty} \frac{\left(1+\frac{n l}{k}\right)^{\frac{k}{l}}}{e^{n}}\left(1+\frac{n l}{k}\right)^{\frac{2}{p l}-\frac{1}{2 p}} \\
\quad \geq \frac{\left|a_{l}\right|^{n}}{\alpha^{n}} \limsup _{k \rightarrow \infty} \frac{\left(1+\frac{n l}{k}\right)^{\frac{k}{l}}}{e^{n}}=\frac{\left|a_{l}\right|^{n}}{\alpha^{n}},
\end{aligned}
$$

which yields $\alpha \geq\left|a_{l}\right|$.

(v) Suppose that $V_{g_{l}}$ is power bounded and uniformly mean ergodic. It follows from [12] that the spectrum $\sigma\left(V_{g_{l}}\right)=\left\{\lambda \in \mathbb{C}:|\lambda| \leq \frac{\left|a_{l}\right|}{\alpha}\right\}$. Hence, if $\left|a_{l}\right|=\alpha$, then 1 is an accumulation point of $\sigma\left(V_{g_{l}}\right)$. By [15, Theorem 3.16] (see also [18, Theorem 2.7]), the operator $V_{g_{l}}$ is not uniformly mean ergodic. This implies $\left|a_{l}\right|<\alpha$.

Corollary 3.4. Let $1 \leq p \leq \infty$ and $m \geq 1$. Then the integration operator $J$ on $\mathcal{F}_{(\alpha, m)}^{p}$ satisfies

(i) If $m>1$ and $1 \leq p \leq \infty$, then $J$ is compact, quasi-nilpotent, hence power bounded, and uniformly mean ergodic on $\mathcal{F}_{(\alpha, m)}^{p}$.

(ii) If $m=1$ and $p=\infty$, then $J$ is power bounded if and only if $\alpha \geq 1$.

(iii) If $m=1$ and $p=\infty$, then $J$ is uniformly mean ergodic if and only if $\alpha>1$.

(iv) If $m=1$ and $1 \leq p<\infty$ and $J$ is power bounded, then $\alpha \geq 1$.

(v) If $m=1$ and $1 \leq p<\infty$ and $J$ is power bounded and uniformly mean ergodic, then $\alpha>1$.

Proof. This follows immediately from Theorem 3.3.

\section{Dynamics of the Hardy operator on $\mathcal{F}_{(\alpha, m)}^{p}$}

In this section we study the dynamics of the classical Hardy operator $H f(z)=$ $\frac{1}{z} \int_{0}^{z} f(w) d w=\frac{1}{z} J f(z)$ on the spaces $\mathcal{F}_{(\alpha, m)}^{p}$. Dynamical properties of this operator has been investigated in related contexts in $[5,6]$.

Theorem 4.1. Let $1 \leq p<\infty$. Then the Hardy operator $H$ is both power bounded and uniformly mean ergodic on $\mathcal{F}_{(\alpha, m)}^{p}$. Furthermore, $\|H\|=1$.

Proof. Let us first show that $H$ is bounded and $\|H\|=1$. Proceeding as in [5], we get

$$
M_{p}^{p}(H f, r) \leq M_{p}^{p}(f, r)
$$


Multiplying both sides by $2 \pi r e^{-p \alpha r^{m}}$ and integrating over $r$ yields

$$
\|H f\|_{(p, \alpha, m)}^{p} \leq\|f\|_{(p, \alpha, m)}^{p},
$$

which implies that $H$ is bounded and $\|H\| \leq 1$.

On the other hand, if $H$ is bounded, then

$$
\|H \mathbf{1}\|_{(p, \alpha, m)}^{p}=\int_{\mathbb{C}}|H \mathbf{1}(z)|^{p} e^{-p \alpha|z|^{m}} d A(z)=\|\mathbf{1}\|_{(p, \alpha, m)}^{p}
$$

and hence $\|H\|=1$.

Next fix $n>1$. Let $f(z)=\sum_{k=0}^{\infty} a_{k} z^{k}$ be the Taylor series expansion of $f \in \mathcal{F}_{(\alpha, m)}^{p}$. A simple integral computation shows that

$$
H^{n} f(z)=\sum_{k=0}^{\infty} \frac{a_{k} z^{k}}{(k+1)^{n}} .
$$

On the other hand, for each $r>0$, applying Cauchy inequalities

$$
\left|a_{k}\right| r^{k}=\frac{r^{k}}{2 \pi}\left|\int_{|\zeta|=r} \frac{|f(\zeta)|}{\zeta^{k+1}} d \zeta\right| \leq \frac{1}{2 \pi} \int_{0}^{2 \pi} \mid f\left(r e^{i \theta)} \mid d \theta \leq M_{p}(f, r),\right.
$$

from which we get $\left|a_{k}\right|\left\|z^{k}\right\|_{(p, \alpha, m)} \leq\|f\|_{(p, \alpha, m)}$ holds for $k \geq 0$. This and (4.1) imply

$$
\left\|H^{n} f\right\|_{(p, \alpha, m)} \leq \sum_{k=0}^{\infty} \frac{\left|a_{k}\right|\left\|z^{k}\right\|_{(p, \alpha, m)}}{(k+1)^{n}} \leq\|f\|_{(p, \alpha, m)} \sum_{k=0}^{\infty} \frac{1}{(k+1)^{n}} \simeq \frac{\|f\|_{(p, \alpha, m)}}{n-1}
$$

for all $n>1$. Therefore, $H$ is power bounded. Observe also that,

$$
\begin{aligned}
\left\|\frac{1}{n} \sum_{j=1}^{n} H^{j}\right\| \leq \frac{1}{n} \sum_{j=1}^{n}\left\|H^{j}\right\| & =\frac{1}{n}\left(\|H\|+\sum_{j=2}^{n}\left\|H^{j}\right\|\right)=\frac{1}{n}+\frac{1}{n} \sum_{j=2}^{n}\left\|H^{j}\right\| \\
& \lesssim \frac{1}{n}+\frac{1}{n} \sum_{j=2}^{n} \frac{1}{j-1} \simeq \frac{1}{n}+\frac{\log |n-1|}{n} \rightarrow 0
\end{aligned}
$$

as $n \rightarrow \infty$. Therefore, $H$ is also uniformly mean ergodic, which completes the proof.

We now mention consequences of Theorem 4.1. Since all orbits $\left\{T^{n} f ; n=\right.$ $0,1,2, \ldots\}$ of any power bounded operator $T$ are bounded, it cannot be hypercyclic. This conclusion fails to hold for the supercyclicity property in general. But if the operator satisfies in addition for example $T f(\zeta)=f(\zeta)$ for all $f$ in the space and at least one point $\zeta \in \mathbb{C}$, then $T$ is not supercyclic either. We will prove this for the operator $H$, and the same proof works in general for any other power bounded operator $T$.

Corollary 4.2. Let $1 \leq p<\infty$. Then the Hardy operator $H$ is not supercyclic on $\mathcal{F}_{(\alpha, m)}^{p}$.

Proof. For each $f$ and each $n$ we have

$$
H f(0)=f(0)=H^{n} f(0) .
$$


Proceeding by contradiction, suppose that $f$ is a supercyclic vector for $H$. Then for the constant function $\mathbf{1}$, there exits a sequence $\left(\lambda_{k} H^{n_{k}} f\right)$ in the projective orbit such that $\lambda_{k} H^{n_{k}} f \rightarrow \mathbf{1}$ as $k \rightarrow \infty$. Consequently, by (4.2),

$$
\lim _{k \rightarrow \infty} \lambda_{k} H^{n_{k}} f(0)=\lim _{k \rightarrow \infty} \lambda_{k} f(0)=1
$$

hence $f(0) \neq 0$ and $\left(\lambda_{k}\right)$ is not a null sequence either.

Similarly, for the function $h(z)=z$, there exists again a sequence $\left(\theta_{j} H^{n_{j}} f\right)$ in the projective orbit such that $\theta_{j} H^{n_{j}} f \rightarrow h$ as $j \rightarrow \infty$, and

$$
\begin{aligned}
& \lim _{j \rightarrow \infty} \theta_{j} H^{n_{j}} f(0)=\lim _{j \rightarrow \infty} \theta_{j} f(0)=h(0)=0 \\
& \lim _{j \rightarrow \infty} \theta_{j} H^{n_{j}} f(1)=\lim _{j \rightarrow \infty} \theta_{j} f(1)=h(1)=1,
\end{aligned}
$$

from which, since $f(0) \neq 0$, we deduce $\theta_{j} \rightarrow 0$ as $j \rightarrow \infty$. This and power boundedness in Theorem 4.1 yield

$$
\lim _{j \rightarrow \infty}\left\|\theta_{j} H^{n_{j}} f\right\|_{(p, \alpha, m)} \lesssim\|f\|_{(p, \alpha, m)} \lim _{j \rightarrow \infty}\left|\theta_{j}\right|=0 .
$$

Therefore, $\theta_{j} H^{n_{j}} f \rightarrow \mathbf{0}$ which implies $\theta_{j} H^{n_{j}} f(1) \rightarrow 0$ as $j \rightarrow \infty$. This contradicts (4.3).

\section{The RitT's RESOlvent CONDITION}

A classical operator theoretic problem, for any given bounded operator $T$ on a complex Banach space $X$, is to identify the relation between the size of the resolvent $(T-\lambda I)^{-1}$ when $\lambda$ is near to the spectrum of $T$ and the asymptotic behaviour of the orbits $\left\{T^{n} x: x \in X\right\}$. In this perspective, recall that the Ritt's condition [27] for $T$ states that there exists a positive constant $C$ such that

$$
\left\|(T-\lambda I)^{-1}\right\| \leq \frac{C}{|\lambda-1|}
$$

for each $\lambda \in \mathbb{C}$ and $|\lambda|>1$. As an immediate consequence of Theorem 4.1, it turns out that the Hardy operator $H$ on generalized Fock spaces belongs to the class of operators satisfying such condition.

Proposition 5.1. Let $1 \leq p<\infty$. Then

(i) the Hardy operator $H$ satisfies the Ritt's resolvent condition on $\mathcal{F}_{(\alpha, m)}^{p}$.

(ii) the differentiation operator $D$ satisfies the Ritt's resolvent condition on $\mathcal{F}_{(\alpha, m)}^{p}$ if and only if it is power bounded and uniformly mean ergodic.

Proof. (i) Nagy and Zemanek [25] proved that a bounded operator $T$ on a complex Banach space satisfies the Ritt's resolvent condition if and only if it is power bounded and

$$
\sup _{n \geq 1} n\left\|T^{n+1}-T^{n}\right\|<\infty
$$


Thus, by Theorem 4.1, it is enough to show that the operator $H$ satisfies condition (5.1). To this goal, applying (4.1)

$$
\begin{array}{r}
\left\|H^{n+1} f-H^{n} f\right\|_{(p, \alpha, m)} \leq \sum_{k=0}^{\infty} \frac{\left|a_{k}\right|\left\|z^{k}\right\|_{(p, \alpha, m)}}{(k+1)^{n}}\left|\frac{1}{k+1}-1\right| \\
\leq\|f\|_{(p, \alpha, m)} \sum_{k=0}^{\infty} \frac{k}{(k+1)^{n+1}} \simeq \frac{\|f\|_{(p, \alpha, m)}}{n},
\end{array}
$$

and the conclusion easily follows.

(ii) Assume first that $D$ is power bounded and uniformly mean ergodic. Then by Theorem 2.2, either $m<1$ or $m=1$ and $\alpha<1$. For $m<1$, arguing as in (2.1) there exist $N \in \mathbb{N}$ and $0<\delta<1$ such that

$$
n\left\|D^{n+1}-D^{n}\right\| \leq n\left\|D^{n+1}\right\|+n\left\|D^{n}\right\| \leq 2 n \delta^{n} \text { for all } n \geq N .
$$

Similarly, if $m=1$ and $\alpha<1$, then (2.2) implies

$$
n\left\|D^{n+1}-D^{n}\right\| \leq n\left\|D^{n+1}\right\|+n\left\|D^{n}\right\| \leq n \frac{(n+1)^{\frac{p}{2}}}{e^{(1-\alpha)(n+1)}}+n \frac{n^{\frac{p}{2}}}{e^{(1-\alpha) n}} .
$$

Now we take the supremum with respect to $n \in \mathbb{N}$ both in (5.2) and (5.3) to see that condition (5.1) is satisfied.

For the other implication, by a result of Nagy and Zemanek [25], it is enough to show that $D$ is uniformly mean ergodic. We arrive at this conclusion if we show that the Ritt's condition fails when $m=1$ and $\alpha \geq 1$. If $m=1$, then using again the exponential functions, $e_{\beta}(z)=e^{\beta z},|\beta|<\alpha$, we get $\overline{D(0, \alpha)} \subset \sigma(D)$. Thus, then the spectrum $\sigma(D)$ contains the unit circle $\mathbb{T}$ whenever if $\alpha \geq 1$. This is a contradiction since the spectrum of an operator which satisfies the Ritt's resolvent condition contains only 1 from the unit circle; see [19] and [26, Theorem 4.5.4] for more details.

Acknowledgement. The authors are very thankful to the referee for the careful reading of our paper and many suggestions which corrected and improved our manuscript.

Part of this work was done during the third-named author's stay at the Instituto Universitario de Matemática Pura y Aplicada of the Universitat Politècnica de València. He would like to thank Prof. José Bonet, Prof. Alfred Peris and all other members of the institute for their hospitality and kindness during his stay in Valencia, Spain.

\section{REFERENCES}

[1] A. V. Abanin, P. T. Tien, Differentiation and integration operators on weighted Banach spaces of holomorphic functions, Math. Nachr., 290(2017), no. 8-9, 11441162 .

[2] A. Atzmon, B. Brive, Surjectivity and invariant subspaces of differential operators on weighted Bergman spaces of entire functions, Bergman spaces and related topics in complex analysis, Contemp. Math., vol. 404, Amer. Math. Soc., Providence, RI, 2006, 27-39. 
[3] F. Bayart, E. Matheron, Dynamics of linear operators, Cambridge Tracts in Math. 179. Cambridge Univ. Press, Cambridge, 2009.

[4] T. Bermúdez, A. Bonilla, A. Peris, On hypercyclicity and supercyclicity criteria, Bull. Austral. Math. Soc., 70 (2004), 45-54.

[5] M. J. Beltrán, Dynamics of differentiation and integration operators on weighted space of entire functions, Studia Math., 221 (2014), 35-60.

[6] M. J. Beltrán, J. Bonet, C. Fernández, Classical operators on weighted Banach spaces of entire functions, Proc. Amer. Math. Soc., 141 (2013), 4293-4303.

[7] J. Bès, A. Peris, Hereditarily hypercyclic operators, J. Funct. Anal., 167 (1999), 94-112.

[8] J. Bonet, Dynamics of the differentiation operator on weighted spaces of entire functions, Math. Z., 26 (2009), 649-657.

[9] J. Bonet, The spectrum of Volterra operators on weighted Banach spaces of entire functions, Quart. J. Math., 66 (2015), 799-807.

[10] J. Bonet, A. Bonilla, Chaos of the Differentiation Operator on Weighted Banach Spaces of Entire Functions, Complex Anal. Oper. Theory, 7 (2013), 33-42.

[11] J. Bonet, J. Taskinen, A note about Volterra operators on weighted Banach spaces of entire functions, Math. Nachr. 288 (2015), 1216-1225.

[12] O. Constantin, A.-M. Persson, The spectrum of Volterra-type integration operators on generalized Fock spaces, Bull. London Math. Soc., 47 (2015), 958-963.

[13] O. Constantin, J.-Á. Peláez, Integral Operators, Embedding Theorems and a Littlewood-Paley Formula on Weighted Fock Spaces, J. Geom. Anal., 26 (2016), 1109-1154.

[14] M. De La Rosa, C. Read, A hypercyclic operator whose direct sum is not hypercyclic, J. Operator Theory, 61 (2009), 369-380.

[15] N. Dunford, Spectral theory. I. Convergence to projections. Trans. Amer. Math. Soc., 54 (1943), 185-217.

[16] K. G. Grosse-Erdmann, A. Peris Manguillot, Linear Chaos, Springer, New York, 2011.

[17] A. Harutyunyan, W. Lusky, On the boundedness of the differentiation operator between weighted spaces of holomorphic functions, Studia Math., 184 (2008), 233247.

[18] U. Krengel, Ergodic Theorems, Walter de Gruyter, Berlin, 1985.

[19] Yu. Lyubich. Spectral localization, power boundedness and invariant subspaces under Ritt's type condition. Studia Mathematica, 143(2), (1999) 153-167.

[20] T. Mengestie, A note on the differential operator on generalized Fock spaces, J. Math. Anal. Appl., 458 (2018), no. 2, 937-948.

[21] T. Mengestie, Spectral properties of Volterra-type integral operators on FockSobolev spaces, J. Korean Math. Soc., 54 (2017), no. 6, 1801-1816.

[22] T. Mengestie, On the Spectrum of Volterra-Type Integral Operators on FockSobolev Spaces, Complex Anal. Oper. Theory, 11 (2017), no. 6, 1451-1461.

[23] T. Mengestie, S. Ueki, Integral, differential and multiplication operators on weighted Fock spaces, Complex Anal. Oper. Theory, 13 (2019), 935-95.

[24] T. Mengestie, M. Worku, Isolated and essentially isolated Volterra-type integral operators on generalized Fock spaces, Integr. Transf. Special Funct., 30 (2019), 4154.

[25] B. Nagy, J. A. Zemanek, A resolvent condition implying power boundedness, Studia Math., 134 (1999), 143-151.

[26] O. Nevanlinna, Convergence of iterations for linear equations, Lecture Notes in Mathematics, ETH Zürich, Birkhäuser, Basel, 1993.

[27] R. K. Ritt, A condition that $\lim _{n \rightarrow \infty} n^{-1} T^{n}=0$, Proc. Amer. Math. Soc., 4 (1953), 898-899. 
[28] S. Ueki, Characterization for Fock-type space via higher order derivatives and its application. Complex Anal. Oper. Theory, 8 (2014), 1475-1486.

[29] K. Yosida, Functional Analysis, Springer, Berlin, 1978.

[30] K. Yosida, S. Kakutani, Operator-theoretical treatment of Marko's process and mean ergodic theorem, Ann. Math., 42 (1941), no. 1, 188-228.

Instituto Universitario de Matemática Pura y Aplicada iUMPA, Universitat Politècnica de ValÈnCia, E-46071 VAlencia, Spain

E-mail address: jbonet@mat.upv.es

Department of Mathematical Sciences, Western Norway University of ApPlied Sciences, KlingenbergVegen 8, N-5414 Stord, Norway

E-mail address: Tesfa.Mengestie@hvl.no

Department of Mathematics, Addis Ababa University, Ethiopia

E-mail address: mafuzhumer@gmail.com 